\title{
Introduction to regression
}

\author{
Paul Schrimpf
}

UBC

Economics 326

January 23, 2018 


\section{Review of last week}

- Expectations and conditional expectations

- Linear

- Iterated expectations

- Asymptotics - using large sample distribution to approximate finite sample distribution of estimators

- LLN: sample moments converge in probability to population moments,

$$
\underbrace{\frac{1}{n} \sum_{i=1}^{n} g\left(x_{i}\right)}_{\text {sample moment }} \stackrel{p}{\rightarrow} \underbrace{\mathrm{E}[g(x)]}_{\text {population moment }}
$$

- CLT: centered and scaled sample moments converge in distribution to population moments

$$
\underbrace{\sqrt{n}}_{\text {"scaling" }}(\frac{1}{n} \sum_{i=1}^{n} g\left(x_{i}\right) \underbrace{-\mathrm{E}[g(x)]}_{\text {"centering" }}) \stackrel{d}{\rightarrow} N(0, \operatorname{Var}(g(x)))
$$

- Using CLT to calculate p-values 


\section{Definition and interpretation of regression}


(1) Motivation

2 Conditional expectation function

3 Population regression Interpretation

(4) Sample regression

(5) Regression in $\mathrm{R}$ 


\section{References}

- Main texts:

- Angrist and Pischke (2014) chapter 2

- Wooldridge (2013) chapter 2

- Stock and Watson (2009) chapter 4-5

- More advanced:

- Angrist and Pischke (2009) chapter 3 up to and including section 3.1.2 (pages 27-40)

- Bierens (2012)

- Abbring (2001) chapter 3

- Baltagi (2002) chapter 3

- Linton (2017) chapters 16-20, 22

- More introductory:

- Diez, Barr, and Cetinkaya-Rundel (2012) chapter 7 


\title{
Paul Schrimpf
}

Motivation

Conditional

expectation

function

Population

regression

Interpretation

Sample

regression

Regression in

\section{Section 1}

\author{
Motivation
}




\section{General problem}

- Often interested in relationship between two (or more) variables, e.g.

- Wages and education

- Minimum wage and unemployment

- Price, quantity, and product characterics

- Usually have:

(1) Variable to be explained (dependent variable)

2 Explanatory variable(s) or independent variables or covariates

Dependent

Wage

Unemployment

Quantity

$Y$
Independent

Education
Price and product characteristics

- For now agnostic about causality, but $E[Y \mid X]$ usually is not causal 


\section{Example: Growth and GDP}

Motivation

Conditional expectation function

Population regression Interpretation Sample regression

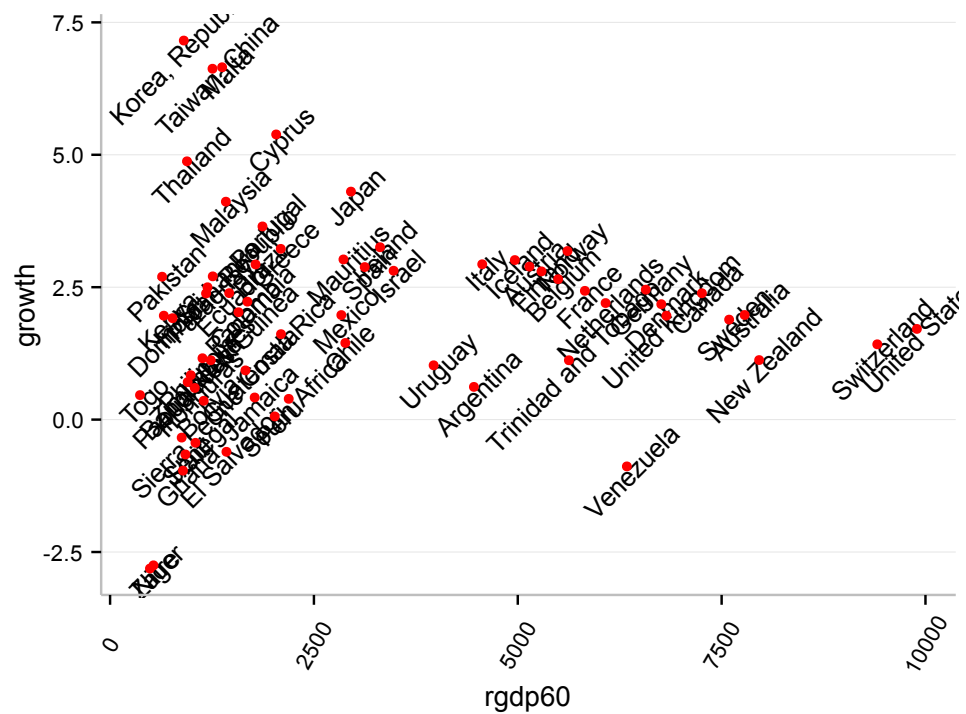




\section{Years of schooling in 1960 and growth}

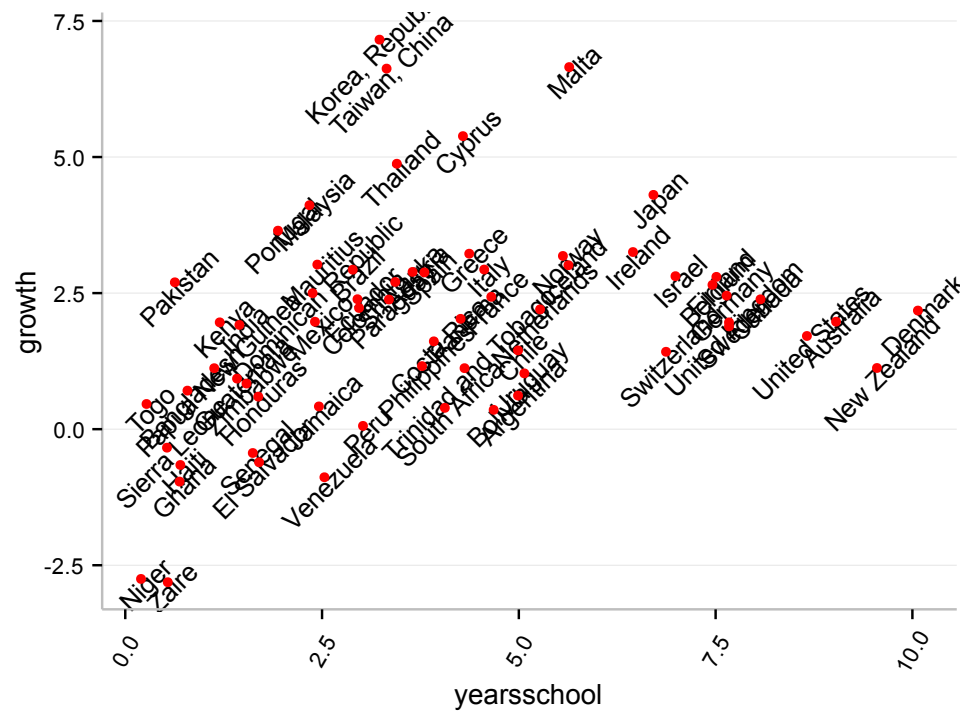




\section{Section 2}

\section{Conditional expectation function}




\section{Conditional expectation}

\section{function}

- One way to describe relation between two variables is a function,

$$
Y=h(X)
$$

- Most relationships in data are not deterministic, so look at average relationship,

$$
\begin{aligned}
Y & =\underbrace{\mathrm{E}[Y \mid X]}_{\equiv h(X)}+\underbrace{(Y-\mathrm{E}[Y \mid X])}_{\equiv \epsilon} \\
& =\mathrm{E}[Y \mid X]+\epsilon
\end{aligned}
$$

- Note that $\mathrm{E}[\epsilon]=0$ (by definition of $\epsilon$ and iterated expectations)

- $E[Y \mid X]$ can be any function, in particular, it need not be linear 


\section{Conditional expectation}

\section{function}

- Unrestricted $\mathrm{E}[Y \mid X]$ hard to work with

- Hard to estimate

- Hard to communicate if $X$ a vector (cannot draw graphs)

- Instead use linear regression

- Easier to estimate and communicate

- Tight connection to $E[Y \mid X]$ 


\section{Section 3}

\section{Population regression}




\section{Population regression}

- The bivariate population regression of $Y$ on $X$ is

$$
\left(\beta_{0}, \beta_{1}\right)=\underset{b_{0}, b_{1}}{\arg \min } \mathrm{E}\left[\left(Y-b_{0}-b_{1} X\right)^{2}\right]
$$

i.e. $\beta_{0}$ and $\beta_{1}$ are the slope and intercept that minimize the expected square error of $Y-\left(\beta_{0}+\beta_{1} X\right)$

- Calculating $\beta_{0}$ and $\beta_{1}$ :

- First order conditions:

$$
\begin{aligned}
{\left[b_{0}\right]: 0 } & =\frac{\partial}{\partial b_{0}} \mathrm{E}\left[\left(Y-b_{0}-b_{1} X\right)^{2}\right] \\
& =\mathrm{E}\left[\frac{\partial}{\partial b_{0}}\left(Y-b_{0}-b_{1} X\right)^{2}\right] \\
& =\mathrm{E}\left[-2\left(Y-\beta_{0}-\beta_{1} X\right)\right]
\end{aligned}
$$


and

\section{Population regression}

Motivation

- (1) rearranged gives $\beta_{0}=\mathrm{E}[Y]-\beta_{1} \mathrm{E}[X]$

- Substituting into (2)

$$
\begin{aligned}
0 & =\mathrm{E}\left[X\left(-Y+\mathrm{E}[Y]-\beta_{1} \mathrm{E}[X]+\beta_{1} X\right)\right] \\
& =\mathrm{E}[X(-Y+\mathrm{E}[Y])]+\beta_{1} \mathrm{E}[X(X-\mathrm{E}[X])] \\
& =-\operatorname{Cov}(X, Y)+\beta_{1} \operatorname{Var}(X) \\
\beta_{1} & =\frac{\operatorname{Cov}(X, Y)}{\operatorname{Var}(X)} \\
-\beta_{1}=\frac{\operatorname{Cov}(X, Y)}{\operatorname{Var}(X)}, \beta_{0} & =\mathrm{E}[Y]-\beta_{1} \mathrm{E}[X]
\end{aligned}
$$




\section{Population regression approximates $\mathrm{E}[Y \mid X]$}

\section{Lemma}

The population regression is the minimal mean square error linear approximation to the conditional expectation function, i.e.

$$
\underbrace{\underset{b_{0}, b_{1}}{\arg \min } \mathrm{E}\left[\left(Y-\left(b_{0}+b_{1} X\right)\right)^{2}\right]}_{\text {population regression }}=\underset{b_{0}, b_{1}}{\arg \min } \underbrace{E_{X}\left[\left(\mathrm{E}[Y \mid X]-\left(b_{0}+b_{1} X\right)\right)^{2}\right]}_{\text {MSE of linear approximation to } \mathrm{E}[Y \mid X]}
$$

\section{Corollary}

If $\mathrm{E}[Y \mid X]=c+m X$, then the population regression of $Y$ on $X$ equals $\mathrm{E}[Y \mid X]$, i.e. $\beta_{0}=c$ and $\beta_{1}=m$ 


\section{Proof.}

- Let $b_{0}^{*}, b_{1}^{*}$ be minimizers of MSE of approximation to $\mathrm{E}[Y \mid X]$

- Same steps as in population regression formula gives

$$
0=\mathrm{E}\left[-2\left(\mathrm{E}[Y \mid X]-b_{0}^{*}-b_{1}^{*} X\right)\right]
$$

and

$$
0=\mathrm{E}\left[-2\left(\mathrm{E}[Y \mid X]-b_{0}^{*}-b_{1}^{*} X\right) X\right]
$$

- Rearranging and combining,

$$
b_{0}^{*}=\mathrm{E}[\mathrm{E}[Y \mid X]]-b_{1}^{*} \mathrm{E}[X]=\mathrm{E}[Y]-b_{1}^{*} \mathrm{E}[X]
$$

and

$$
\begin{aligned}
0 & =\mathrm{E}\left[X\left(-\mathrm{E}[Y \mid X]+\mathrm{E}[Y]+b_{1}^{*} \mathrm{E}[X]-b_{1}^{*} X\right)\right] \\
& =\mathrm{E}[X(-\mathrm{E}[Y \mid X]+\mathrm{E}[Y])]+b_{1}^{*} \mathrm{E}[X(X-\mathrm{E}[X])] \\
& =-\operatorname{Cov}(X, Y)+b_{1}^{*} \operatorname{Var}(X) \\
b_{1}^{*} & =\frac{\operatorname{Cov}(X, Y)}{\operatorname{Var}(X)}
\end{aligned}
$$




\section{Regression interpretation}

- Regression $=$ best linear approximation to $E[Y \mid X]$

- $\beta_{0} \approx \mathrm{E}[Y \mid X=0]$

- $\beta_{1} \approx \frac{d}{d x} \mathrm{E}[Y \mid X] \approx$ change in average $Y$ per unit change in $X$

- Not necessarily a causal relationship (usually not)

- Always can be viewed as description of data 


\section{Regression with binary $X$}

- Suppose $X$ is binary (i.e. can only be 0 or 1 )

- We know $\beta_{0}+\beta_{1} X=$ best linear approximation to $\mathrm{E}[Y \mid X]$

- $X$ only takes two values, so can draw line connecting $\mathrm{E}[Y \mid X=0]$ and $\mathrm{E}[Y \mid X=1]$, so $\beta_{0}+\beta_{1} X=E[Y \mid X]$

- $\beta_{0}=\mathrm{E}[Y \mid X=0]$

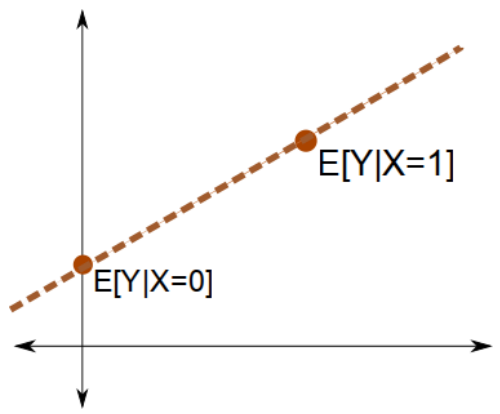

- $\beta_{0}+\beta_{1}=\mathrm{E}[Y \mid X=1]$ 


\section{Section 4}

\section{Sample regression}




\section{Sample regression}

- Have sample of observations: $\left\{\left(y_{i}, x_{i}\right)\right\}_{i=1}^{n}$

- The sample regression (or when unambiguous just "regression") of $Y$ on $X$ is

$$
\left(\hat{\beta}_{0}, \hat{\beta}_{1}\right)=\underset{b_{0}, b_{1}}{\arg \min } \frac{1}{n} \sum_{i=1}^{n}\left(y_{i}-b_{0}-b_{1} x_{i}\right)^{2}
$$

i.e. $\hat{\beta}_{0}$ and $\hat{\beta}_{1}$ are the slope and intercept that minimize the sum of squared errors, $\left(y_{i}-\left(\hat{\beta}_{0}+\hat{\beta}_{1} x_{i}\right)\right)^{2}$

- Same as population regression but with sample average instead of expectation

- Same calculation as for population regression would show

$$
\hat{\beta}_{1}=\frac{\widehat{\operatorname{Cov}}(X, Y)}{\widehat{\operatorname{Var}(X)}}=\frac{\frac{1}{n} \sum_{i=1}^{n}\left(x_{i}-\bar{x}\right)\left(y_{i}-\bar{y}\right)}{\frac{1}{n} \sum_{i=1}^{n}\left(x_{i}-\bar{x}\right)^{2}}
$$

and

$$
\hat{\beta}_{0}=\bar{y}-\hat{\beta}_{1} \bar{x}
$$




\section{Sample regression}

- Sample regression is an estimator for the population regression

- Given an estimator we should ask:

- Unbiased?

- Variance?

- Consistent?

- Asymptotically normal?

- We will address these questions in the next week or two 


\section{Paul Schrimpf}

Motivation

Conditional

expectation

function

Population

regression

Interpretation

Sample

regression

Regression in $\mathrm{R}$

\section{Section 5}

\section{Regression in $\mathrm{R}$}




\section{Regression in $\mathrm{R}$}

Motivation

Conditional expectation function

Population regression

Interpretation

Sample

regression

Regression in $\mathrm{R}$
1 require(datasets) \#\# some datasets included with $R$

2 stateDF $<-$ data.frame (state.x77)

3 summary(stateDF) \#\# summary statistics of data

4

5 \#\# Sample regression function

6 regress $<-$ function $(y, x)\{$

7 beta $<-$ vector (length $=2)$

8 beta [2] $<-\operatorname{cov}(x, y) / \operatorname{var}(x)$

$9 \quad$ beta [1] $<-\operatorname{mean}(y)-\operatorname{beta}[2] * \operatorname{mean}(x)$

10 return (beta)

$11\}$

12

13 \#\# Regress life expectancy on income

${ }_{14}$ beta - regress (stateDF [, "Life. Exp"], stateDF\$Income)

15 beta

16

17 \#\# builtin regression

${ }_{18} \operatorname{Im}($ Life.Exp Income, data=stateDF)

19 \#\# more detailed output

$20 \operatorname{summary}(\operatorname{Im}($ Life. Exp Income, data=stateDF ))

https://bitbucket.org/paulschrimpf/econ326/src/

master/notes $/ 03 /$ regress. R? at=master 
Introduction

to regression

Paul Schrimpf

Fitted value

and residuals

Statistical

properties

Unbiased

Variance

Distribution

Discussion of

assumptions

Examples

Inference

Examples (continued)

Estimating $\sigma_{f}^{2}$

Confidence intervals

Efficiency

References

\section{Part II}

\section{Properties of regression}


Introduction

to regression

Paul Schrimpf

Fitted value

and residuals

Statistical

properties

Unbiased

Variance

Distribution

Discussion of

assumptions

Examples

Inference

Examples (continued)

Estimating $\sigma_{f}^{2}$

Confidence intervals

Efficiency

References

\section{Section 6}

\section{Fitted value and residuals}




\section{Fitted values and residuals}

Fitted value and residuals

Statistical

properties

Unbiased

Variance

Distribution

Discussion of

assumptions

Examples

Inference

Examples (continued) Estimating $\sigma_{\epsilon}^{2}$

- Fitted values:

$$
\hat{y}_{i}=\hat{\beta}_{0}+\hat{\beta}_{1} x_{i}
$$

- Residuals:

$$
\begin{gathered}
\hat{\epsilon}_{i}=y_{i}-\hat{\beta}_{0}-\hat{\beta}_{1} x_{i}=y_{i}-\hat{y}_{i} \\
y_{i}=\hat{y}_{i}+\hat{\epsilon}_{i}
\end{gathered}
$$

- Sample mean of residuals $=0$

- First order condition for $\hat{\beta}_{0}$,

$$
\begin{aligned}
& 0=\frac{1}{n} \sum_{i=1}^{n}\left(y_{i}-\hat{\beta}_{0}-\hat{\beta}_{1} x_{i}\right) \\
& 0=\frac{1}{n} \sum_{i=1}^{n} \hat{\epsilon}_{i}
\end{aligned}
$$

- Sample covariance of $x$ and $\hat{\epsilon}=0$ 


\section{Fitted values and residuals}

- First order condition for $\hat{\beta}_{1}$,

$$
\begin{aligned}
& 0=\frac{1}{n} \sum_{i=1}^{n}\left(y_{i}-\hat{\beta}_{0}-\hat{\beta}_{1} x_{i}\right) x_{i} \\
& 0=\frac{1}{n} \sum_{i=1}^{n} \hat{\epsilon}_{i} x_{i}
\end{aligned}
$$




\section{Fitted values and residuals}

Fitted value and residuals

properties

Unbiased

Variance

Distribution

Discussion of

assumptions

Examples

Inference

Examples (continued) Estimating $\sigma_{\epsilon}^{2}$

Confidence intervals

Efficiency

References

- Sample mean of $\hat{y}_{i}=\bar{y}=\hat{\beta}_{0}+\hat{\beta}_{1} \bar{x}$

$$
\begin{aligned}
\frac{1}{n} \sum_{i=1}^{n} y_{i} & =\frac{1}{n} \sum_{i=1}^{n} \hat{y}_{i}+\hat{\epsilon}_{i} \\
& =\frac{1}{n} \sum_{i=1}^{n} \hat{y}_{i} \\
& =\frac{1}{n} \sum_{i=1}^{n} \hat{\beta}_{0}+\hat{\beta}_{1} x_{i} \\
& =\hat{\beta}_{0}+\hat{\beta}_{1} \bar{x}
\end{aligned}
$$




\section{Fitted values and residuals}

Fitted value and residuals

Statistical

properties

Unbiased

Variance

Distribution

Discussion of

assumptions

Examples

Inference

Examples (continued) Estimating $\sigma_{f}^{2}$

Confidence intervals

Efficiency

- Sample covariance of $y$ and $\hat{\epsilon}=$ sample variance of $\hat{\epsilon}$ :

$$
\begin{aligned}
\frac{1}{n} \sum_{i=1}^{n} y_{i}\left(\hat{\epsilon}_{i}-\overline{\hat{\epsilon}}\right) & =\frac{1}{n} \sum_{i=1}^{n} y_{i} \hat{\epsilon}_{i} \\
& =\frac{1}{n} \sum_{i=1}^{n}\left(\hat{\beta}_{0}+\hat{\beta}_{1} x_{i}+\hat{\epsilon}_{i}\right) \hat{\epsilon}_{i} \\
& =\hat{\beta}_{0} \frac{1}{n} \sum_{i=1}^{n} \hat{\epsilon}_{i}+\beta_{1} \frac{1}{n} \sum_{i=1}^{n} x_{i} \hat{\epsilon}_{i}+\frac{1}{n} \sum_{i=1}^{n} \hat{\epsilon}_{i}^{2} \\
& =\frac{1}{n} \sum_{i=1}^{n} \hat{\epsilon}_{i}^{2}
\end{aligned}
$$


- Decompose $y_{i}$

$$
y_{i}=\hat{y}_{i}+\hat{\epsilon}_{i}
$$

- Total sum of squares $=$ explained sum of squares + sum of squared residuals

$$
\underbrace{\frac{1}{n} \sum_{i=1}^{n}\left(y_{i}-\bar{y}\right)^{2}}_{\text {SST }}=\underbrace{\frac{1}{n} \sum_{i=1}^{n}\left(\hat{y}_{i}-\bar{y}\right)^{2}}_{\text {SSE }}+\underbrace{\frac{1}{n} \sum_{i=1}^{n} \hat{\epsilon}_{i}^{2}}_{S S R}
$$

- R-squared: fraction of sample variation in $y$ that is explained by $x$

$$
R^{2}=\frac{S S E}{S S T}=1-\frac{S S R}{S S T}=\widehat{o \operatorname{Corr}}(y, \hat{y})
$$

- $0 \leq R^{2} \leq 1$

- If all data on regression line, then $R^{2}=1$

- Magnitude of $R^{2}$ does not have direct bearing on economic importance of a regression 
Introduction

to regression

Paul Schrimpf

Fitted value

and residuals

Statistical

properties

Unbiased

Variance

Distribution

Discussion of

assumptions

Examples

Inference

Examples (continued)

Estimating $\sigma_{f}^{2}$

Confidence intervals

Efficiency

References

\section{Section 7}

\section{Statistical properties}




\section{Unbiased}

- $\mathrm{E}[\hat{\beta}]=$ ?

- Assume:

SLR.1 (linear model) $y_{i}=\beta_{0}+\beta_{1} x_{i}+\epsilon_{i}$

SLR.2 (independence) $\left\{\left(x_{i}, y_{i}\right)\right\}_{i=1}^{n}$ is independent random sample

SLR.3 (rank condition) $\widehat{\operatorname{Var}}(X)>0$

SLR.4 (exogeneity) $\mathrm{E}[\epsilon \mid X]=0$

- Then, $\mathrm{E}\left[\hat{\beta}_{1}\right]=\beta_{1}$ and $\mathrm{E}\left[\hat{\beta}_{0}\right]=\beta_{0}$ 


\section{Variance}

- $\operatorname{Var}(\hat{\beta}) ?$

- Assume SLR.1-4 and

SLR.5 (homoskedasticity) $\operatorname{Var}(\epsilon \mid X)=\sigma^{2}$

- Then,

$$
\operatorname{Var}\left(\hat{\beta}_{1} \mid\left\{x_{i}\right\}_{i=1}^{n}\right)=\frac{\sigma^{2}}{\sum_{i=1}^{n}\left(x_{i}-\bar{x}\right)^{2}}
$$

and

$$
\operatorname{Var}\left(\hat{\beta}_{0} \mid\left\{x_{i}\right\}_{i=1}^{n}\right)=\frac{\sigma^{2} \frac{1}{n} \sum_{i=1}^{n} x_{i}^{2}}{\sum_{i=1}^{n}\left(x_{i}-\bar{x}\right)^{2}}
$$




\section{Distribution with normal errors}

- Assume SLR.1-SLR.5 and SLR. 6 (normality) $\epsilon_{i} \mid x_{i} \sim N\left(0, \sigma^{2}\right)$

- Then $Y \mid X \sim N\left(\beta_{0}+\beta_{1} X, \sigma^{2}\right)$, and

$$
\hat{\beta}_{1} \mid\left\{x_{i}\right\}_{i=1}^{n} \sim N\left(\beta_{1}, \frac{\sigma^{2}}{\sum_{i=1}^{n}\left(x_{i}-\bar{x}\right)^{2}}\right)
$$

- Even without assuming normality, the central limit theorem implies $\hat{\beta}$ is asymptotically normal (details in a later lecture) 


\section{Summary}

- Simple linear regression model assumptions:

SLR.1 (linear model) $Y_{i}=\beta_{0}+\beta_{1} x_{i}+\epsilon_{i}$

SLR.2 (independence) $\left\{\left(x_{i}, y_{i}\right)\right\}_{i=1}^{n}$ is independent random sample

SLR.3 (rank condition) $\widehat{\operatorname{Var}}(X)>0$

SLR.4 (exogeneity) $\mathrm{E}[\epsilon \mid X]=0$

SLR.5 (homoskedasticity) $\operatorname{Var}(\epsilon \mid X)=\sigma^{2}$

SLR.6 (normality) $\epsilon_{i} \mid x_{i} \sim N\left(0, \sigma^{2}\right)$

- $\hat{\beta}$ unbiased if SLR.1-SLR.4

- If also SLR.5, then $\operatorname{Var}\left(\hat{\beta}_{1} \mid\left\{x_{i}\right\}_{i=1}^{n}\right)=\frac{\sigma^{2}}{\sum_{i=1}^{n}\left(x_{i}-\bar{\chi}\right)^{2}}$

- If also SLR.6, then $\hat{\beta}_{1} \mid\left\{x_{i}\right\}_{i=1}^{n} \sim N\left(\beta_{1}, \frac{\sigma^{2}}{\sum_{i=1}^{n}\left(x_{i}-\bar{x}\right)^{2}}\right)$ 


\section{Discussion of assumptions}

SLR.1 Having a linear model makes it easier to state the other assumptions, but we could instead start by saying let $\beta_{1}=\frac{\operatorname{Cov}(X, Y)}{\operatorname{Var}(X)}$ and $\beta_{0}=\mathrm{E}[Y]-\beta_{1} \mathrm{E}[X]$ be the population regression coefficients and define $\epsilon_{i}=y_{i}-\beta_{0}-\beta_{1} x_{i}$ 


\section{Discussion of assumptions}

SLR.2 Independent observations is a good assumption for data from a simple random sample

- Common situations where it fails in economics are when we have a time series of observations, e.g. $\left\{\left(x_{t}, y_{t}\right)\right\}_{t=1}^{n}$ could be unemployment and GDP of Canada for many different years; and clustering, e.g. the data could be students test scores and hours studying and our sample consists of randomly chosen courses or schools-students in the same course would not be independent, but across different courses they might be.

- Still have $\mathrm{E}\left[\hat{\beta}_{1}\right]=\beta_{1}$ with non-independent observations as long as $\mathrm{E}\left[\epsilon_{i} \mid x_{1}, \ldots, x_{n}\right]=0$

- The variance of $\hat{\beta}_{1}$ will change with non-independent observations

- Simulation code 


\section{Discussion of assumptions}

SLR. 3 If $\widehat{\operatorname{Var}}(X)=0$, then $\hat{\beta}_{1}$ involves dividing by 0

- If there is no variation in $X$, then we cannot see how $Y$ is related to $X$ 


\section{Discussion of assumptions}

SLR.4 To think about mean independence of $\epsilon$ from $x$ we should have a model motivating the regression

- If the model we want is just a population regression, then automatically $\mathrm{E}[\epsilon X]=0$, and $\mathrm{E}[\epsilon \mid X]=0$ if the conditional expectation function is linear; if conditional expectation nonlinear maybe still a useful approximation

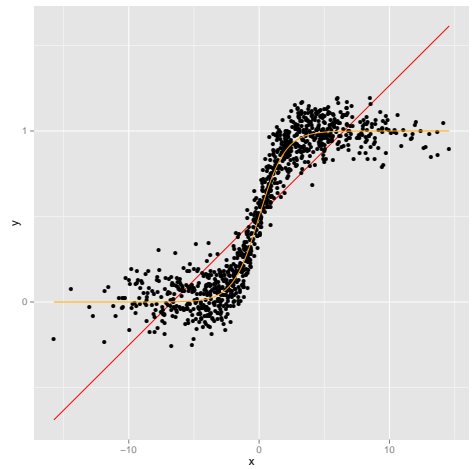




\section{Discussion of assumptions}

SLR.4 To think about mean independence of $\epsilon$ from $x$ we should have a model motivating the regression

- If the model we want is anything else, then maybe $\mathrm{E}[\epsilon X] \neq 0$ (and $\mathrm{E}[\epsilon \mid X] \neq 0)$, e.g.

- Demand curve

$$
p_{i}=\beta_{0}+\beta_{1} q_{i}+\epsilon_{i}
$$

$\epsilon_{i}=$ everything that affects price other than quantity. $q_{i}$ determined in equilibrium implies $\mathrm{E}\left[\epsilon_{i} \mid q_{i}\right] \neq 0$

- $\mathrm{E}\left[\hat{\beta}_{1}\right] \neq \beta_{1}$ and $\hat{\beta}_{1}$

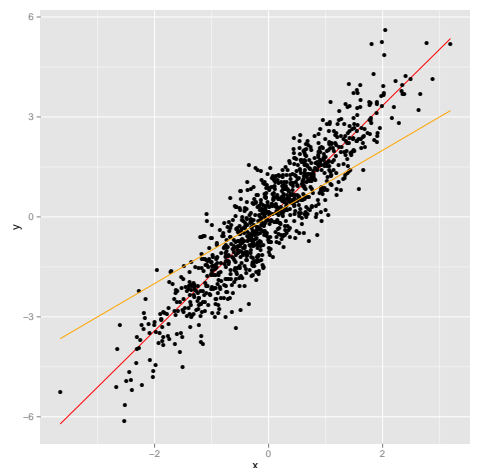
does not tell us what we want 


\section{Discussion of assumptions}

SLR.5 Homoskedasticity: variance of $\epsilon$ does not depend on $X$ Homoskedastic Heteroskedastic
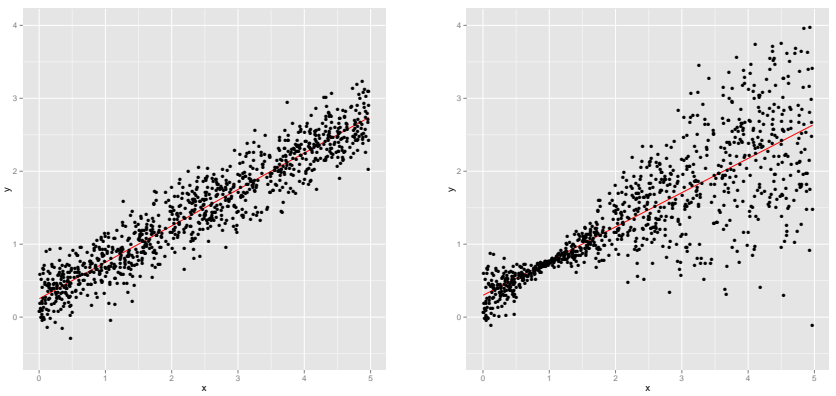

Code

- Heteroskedasticity is when $\operatorname{Var}(\epsilon \mid X)$ varies with $X$

- If there is heteroskedasticity, the variance of $\hat{\beta}_{1}$ is different, but we can fix it 


\section{Discussion of assumptions}

- "robust standard errors" / "heteroscedasticity-consistent (HC) standard errors" / "Eicker-Huber-White standard errors" 


\section{Discussion of assumptions}

\section{SLR.6 If $\epsilon_{i} \mid x_{i} \sim N$, then $\hat{\beta}_{1} \sim N$}

- What if $\epsilon_{i}$ not normally distributed?

- We will see that $\hat{\beta}_{1}$ still asymptotically normal

- Simulation 
Introduction

to regression

Paul Schrimpf

Fitted value

and residuals

Statistical

properties

Unbiased

Variance

Distribution

Discussion of assumptions

Examples

Inference

Examples (continued)

Estimating $\sigma_{f}^{2}$

Confidence intervals

Efficiency

References

\section{Discussion of assumptions}

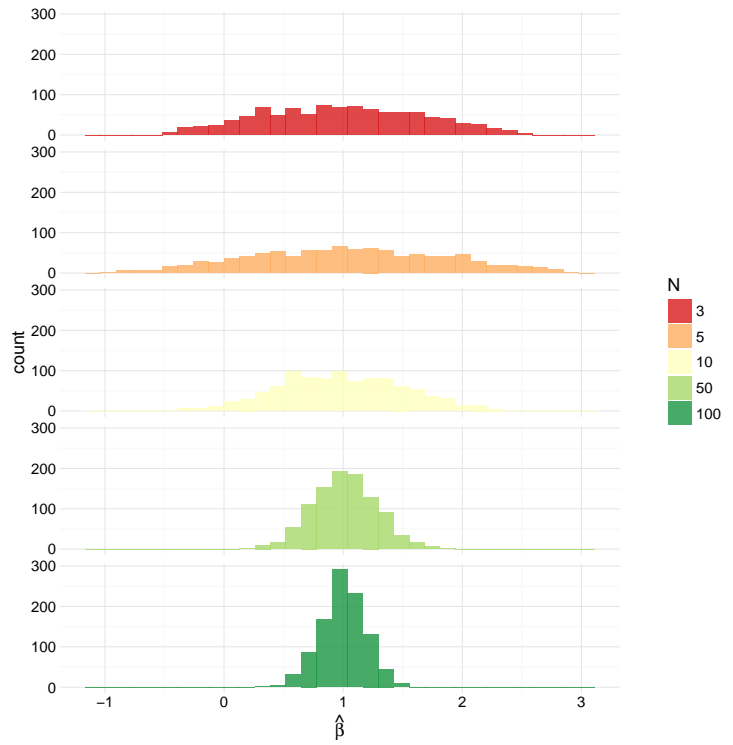


Introduction

to regression

Paul Schrimpf

Fitted value

and residuals

Statistical

properties

Unbiased

Variance

Distribution

Discussion of assumptions

Examples

Inference

Examples (continued) Estimating $\sigma_{c}^{2}$

Confidence intervals

Efficiency

References

\section{Discussion of assumptions}

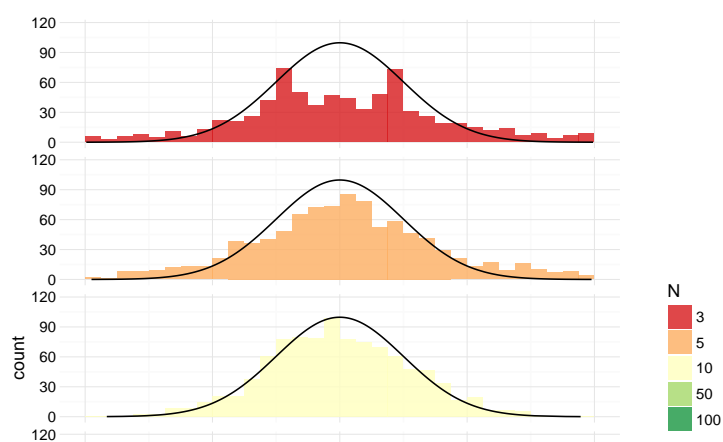




\section{Section 8}

\section{Examples}




\section{Example: smoking and cancer}

- Data on per capita number of cigarettes sold and death rates per thousand from cancer for U.S. states in 1960

- http://lib.stat.cmu.edu/DASL/Datafiles/ cigcancerdat.html

- Death rates from: lung cancer, kidney cancer, bladder cancer, and leukemia Code 
Introduction

to regression

Paul Schrimpf

Fitted value

and residuals

Statistical

properties

Unbiased

Variance

Distribution

Discussion of

assumptions

Examples

Inference

Examples (continued)

Estimating $\sigma_{f}^{2}$

Confidence intervals

Efficiency

References

\section{Smoking and lung cancer}

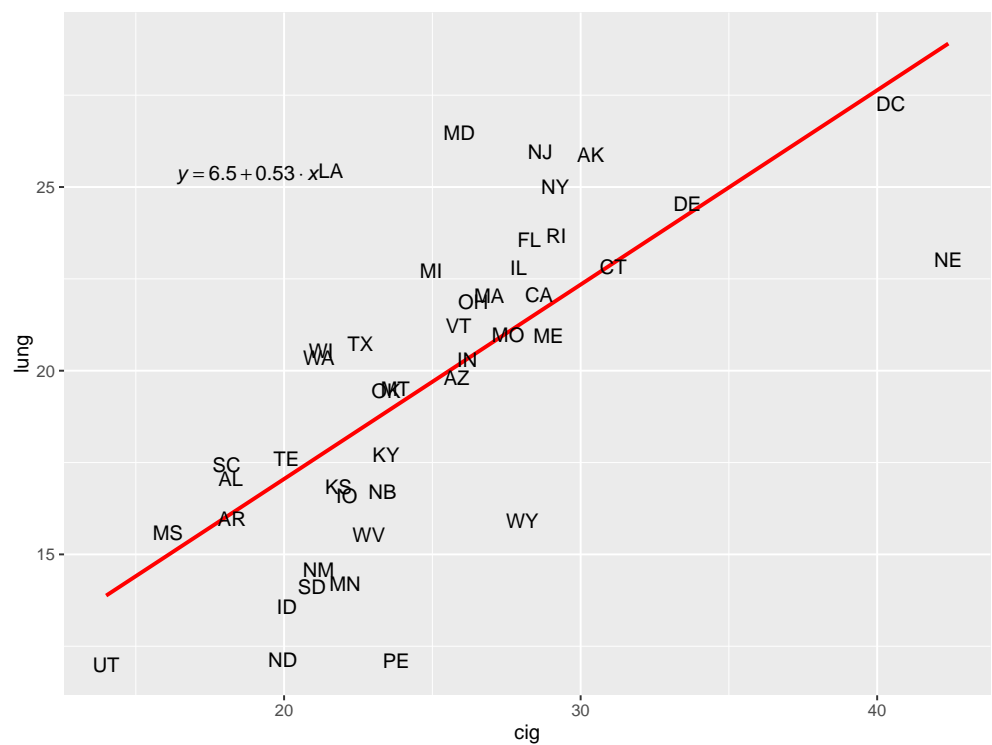


Introduction

to regression

Paul Schrimpf

Fitted value

and residuals

Statistical

properties

Unbiased

Variance

Distribution

Discussion of

assumptions

Examples

Inference

Examples (continued)

Estimating $\sigma_{\epsilon}^{2}$

Confidence intervals

Efficiency

References

\section{Smoking and kidney cancer}

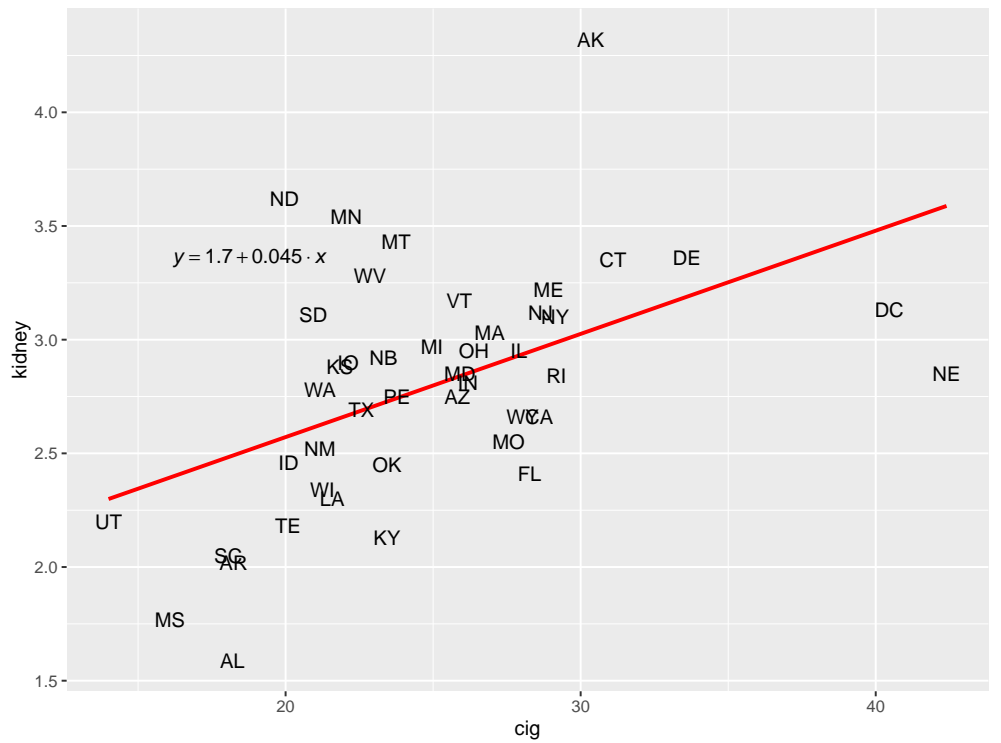


Introduction

to regression

Paul Schrimpf

Fitted value

and residuals

Statistical

properties

Unbiased

Variance

Distribution

Discussion of

assumptions

Examples

Inference

Examples (continued)

Estimating $\sigma_{c}^{2}$

Confidence intervals

Efficiency

References

\section{Smoking and bladder cancer}

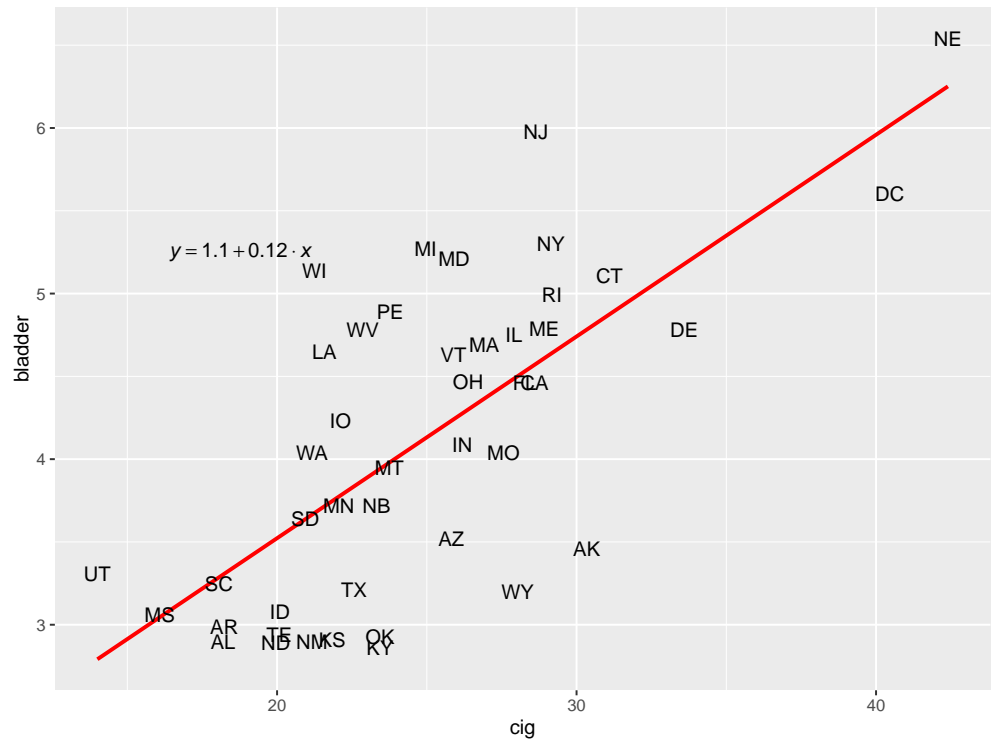


Introduction

to regression

Paul Schrimpf

Fitted value

and residuals

Statistical

properties

Unbiased

Variance

Distribution

Discussion of

assumptions

Examples

Inference

Examples (continued)

Estimating $\sigma_{f}^{2}$

Confidence intervals

Efficiency

References

\section{Smoking and leukemia}

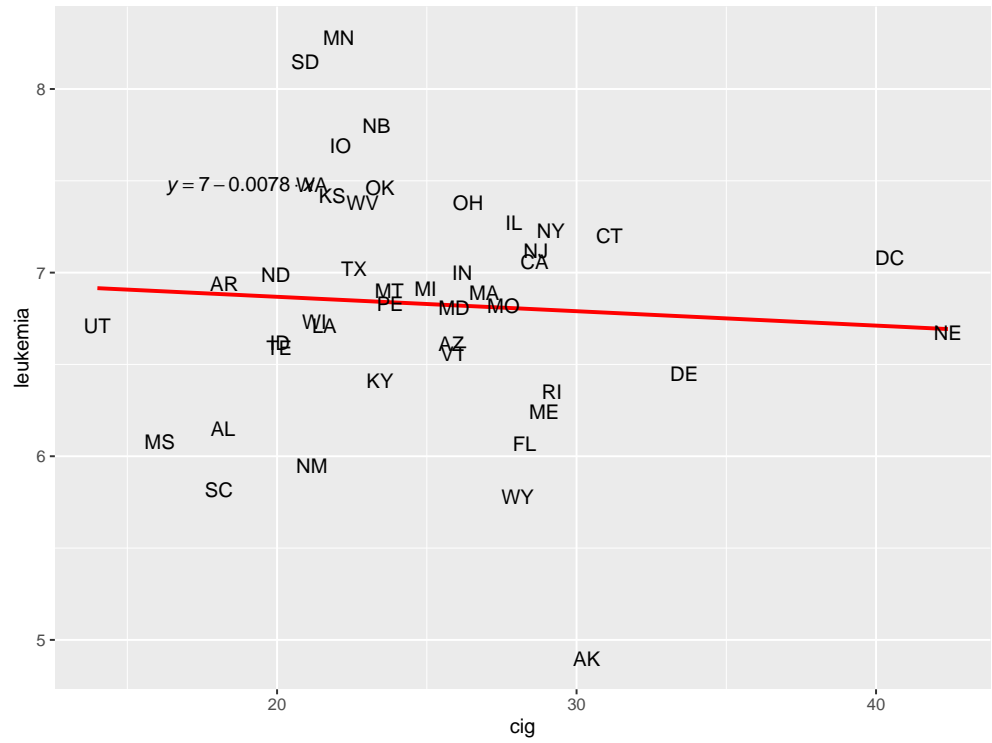




\section{Example: convergence in} growth

- Data on average growth rate from 1960-1995 for 65 countries along with GDP in 1960, average years of schooling in 1960, and other variables

- From http://wps.aw.com/aw_stock_ie_2/50/13016/ 3332253. cw/index.html, originally used in Beck, Levine, and Loayza (2000)

- Question: has there been in convergence, i.e. did poorer countries in 1960 grow faster and catch-up?

- Code 


\section{GDP in 1960 and growth}

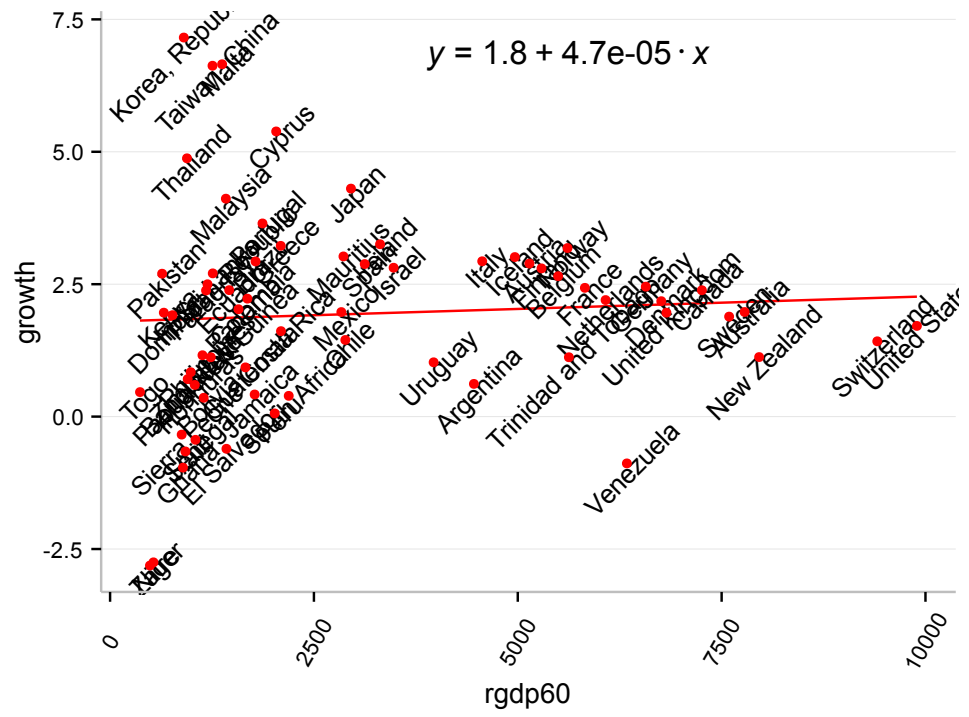




\section{Years of schooling in 1960 and}

\section{growth}

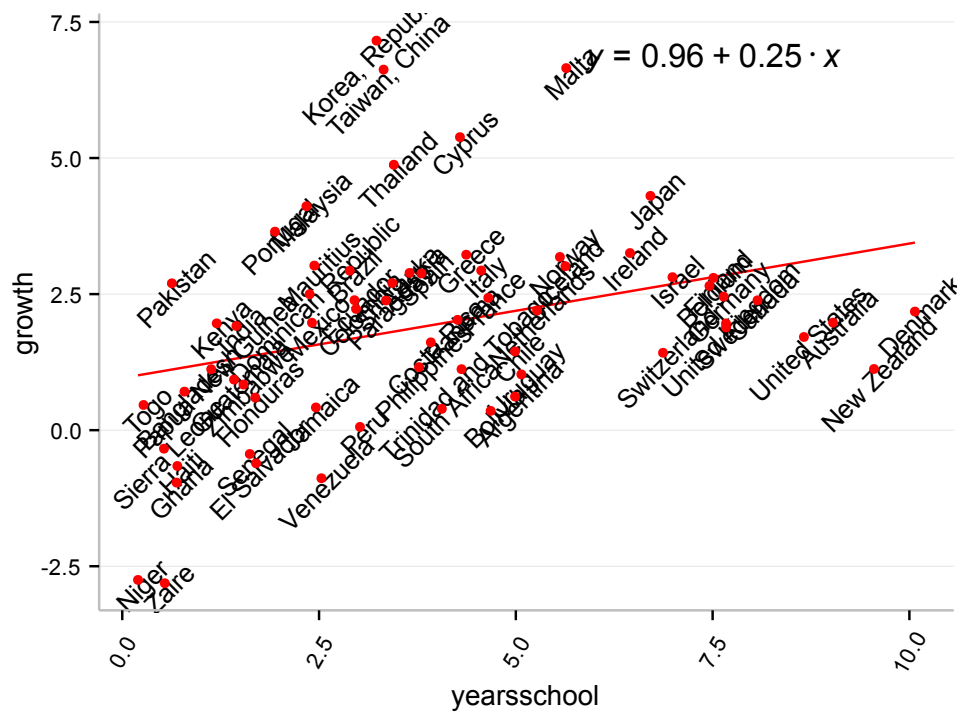


- Things look different 1995-2014

- Code to download and recreate results using updated growth data through 2014 from the World Bank 
Introduction

to regression

Paul Schrimpf

Fitted value

and residuals

Statistical

properties

Unbiased

Variance

Distribution

Discussion of

assumptions

Examples

Inference

Examples (continued)

Estimating $\sigma_{f}^{2}$

Confidence intervals

Efficiency

References

\title{
Section 9
}

\author{
Inference
}




\section{Inference with normal errors}

- Regression estimates depend on samples, which are random, so the regression estimates are random

- Some regressions will randomly look "interesting" due to chance

- Logic of hypothesis testing: figure out probability of getting an interesting regression estimate due solely to change

- Null hypothesis, $H_{0}$ : the regression is uninteresting, usually $\beta_{1}=0$ 


\section{Inference with normal errors}

- With assumptions SR.1-SR.6 and under $H_{0}: \beta_{1}=\beta_{1}^{*}$, we know

$$
\hat{\beta} \sim N\left(\beta_{1}^{*}, \frac{\sigma_{\epsilon}^{2}}{\sum_{i=1}^{n}\left(x_{i}-\bar{x}\right)^{2}}\right)
$$

or equivalently,

$$
t \equiv \frac{\hat{\beta}-\beta_{1}^{*}}{\sigma_{\epsilon} / \sqrt{\sum_{i=1}^{n}\left(x_{i}-\bar{x}\right)^{2}}} \sim N(0,1)
$$

- P-value: the probability of getting a regression estimate as or more "interesting" than the one we have

- As or more interesting $=$ as far or further away from $\beta_{1}^{*}$

- If we are only interested when $\hat{\beta}_{1}$ is on one side of $\beta_{1}^{*}$, then we have a one sided alternative, e.g. $H_{a}: \beta_{1}>\beta_{1}^{*}$

- If we are equally interested in either direction, then $H_{a}: \beta_{1} \neq \beta_{1}^{*}$ 
Introduction

to regression

\section{Paul Schrimpf}

Fitted value and residuals

\section{Statistical}

properties

Unbiased

Variance

Distribution

Discussion of assumptions

\section{Examples}

Inference

Examples (continued)

Estimating $\sigma^{2}$

Confidence intervals

\section{Efficiency}

References

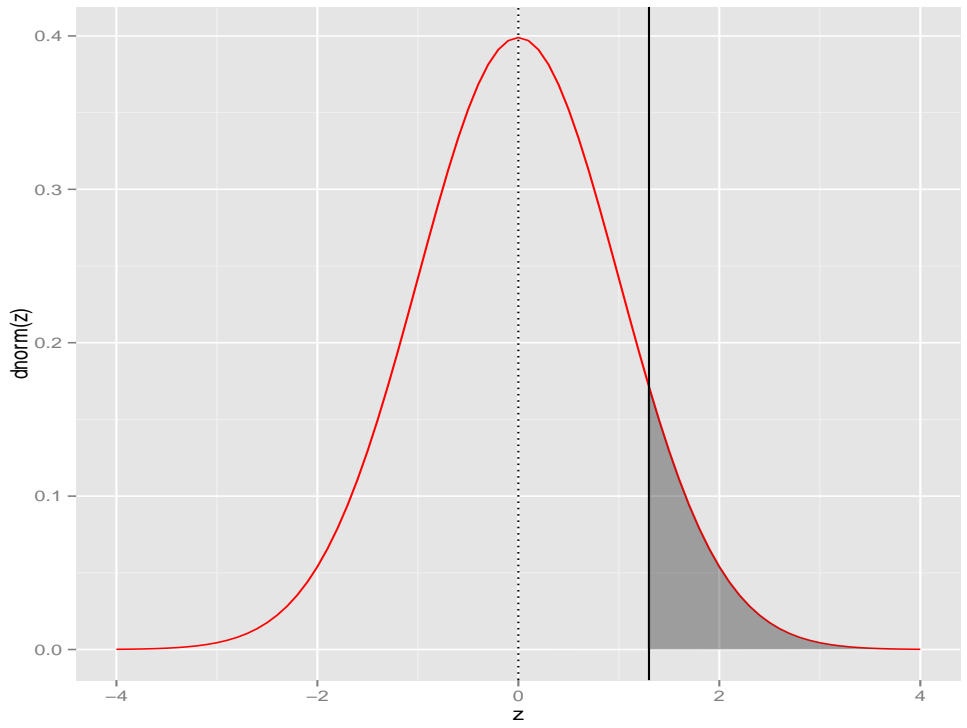


Introduction

to regression

\section{Paul Schrimpf}

Fitted value

and residuals

\section{Statistical}

properties

Unbiased

Variance

Distribution

Discussion of assumptions

\section{Examples}

Inference

Examples (continued)

Estimating $\sigma^{2}$

Confidence intervals

\section{Efficiency}

References

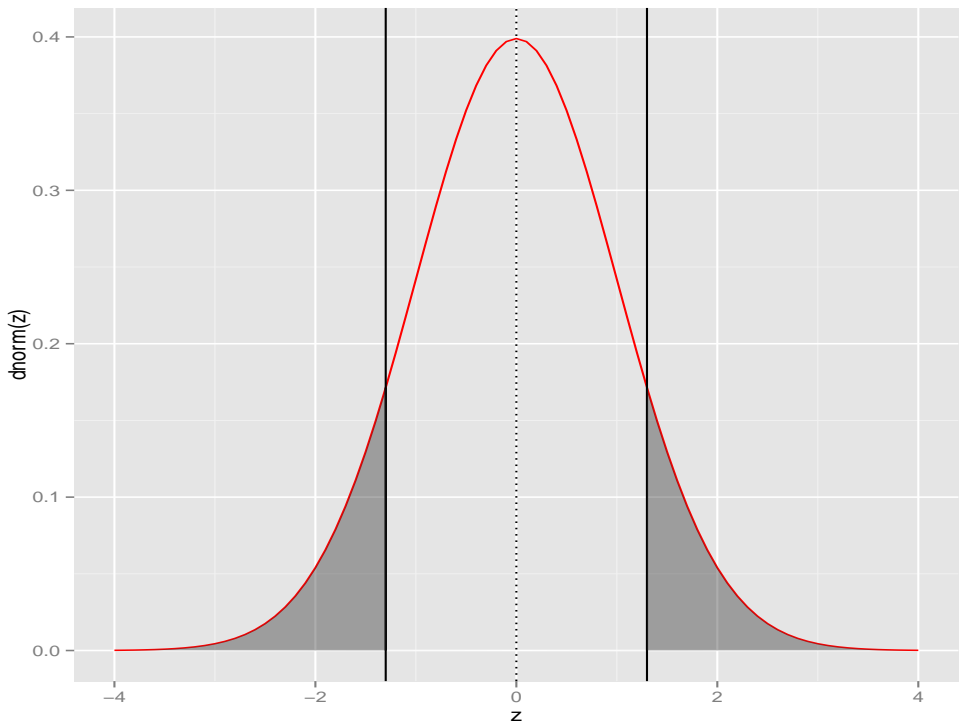




\section{Inference with normal errors}

- One-sided p-value: $p=\Phi(-|t|)=1-\Phi(|t|)$

- Two-sided p-value: $p=2 \Phi(-|t|)=2(1-\Phi(|t|))$

- Interpretation:

- The probability of getting an estimate as strange as the one we have if the null hypothesis is true.

- It is not about the probability of $\beta_{1}$ being any particular value. $\beta_{1}$ is not a random variable. It is some unknown number. The data is what is random. In particular, the p-value is not the probability that that $H_{0}$ is false given the data.

- Hypothesis testing: we must make a decision (usually reject or fail to reject $H_{0}$ )

- Choose significance level $\alpha$ (usually 0.05 or 0.10 )

- Construct procedure such that if $H_{0}$ is true, we will incorrectly reject with probability $\alpha$

- Reject null if $p$-value less than $\alpha$ 


\section{Smoking and cancer}

\begin{tabular}{lcccc}
\hline & Model 1 & Model 2 & Model 3 & Model 4 \\
\hline (Intercept) & $1.09^{*}$ & $6.47^{* *}$ & $1.66^{* * *}$ & $7.03^{* * *}$ \\
& $(0.48)$ & $(2.14)$ & $(0.32)$ & $(0.45)$ \\
cig & $0.12^{* * *}$ & $0.53^{* * *}$ & $0.05^{* * *}$ & -0.01 \\
& $(0.02)$ & $(0.08)$ & $(0.01)$ & $(0.02)$ \\
\hline $\mathrm{R}^{2}$ & 0.50 & 0.49 & 0.24 & 0.00 \\
Adj. R ${ }^{2}$ & 0.48 & 0.47 & 0.22 & -0.02 \\
Num. obs. & 44 & 44 & 44 & 44 \\
RMSE & 0.69 & 3.07 & 0.46 & 0.64 \\
\hline${ }^{* * *} p<0.001,{ }^{* *} p<0.01,{ }^{*} p<0.05$ & & \\
\multicolumn{5}{c}{ Table: Smoking and cancer }
\end{tabular}




\section{Growth and GDP}

\begin{tabular}{lcc}
\hline & Model 1 & Model 2 \\
\hline (Intercept) & $\begin{array}{c}1.80^{* * *} \\
(0.38)\end{array}$ & $\begin{array}{c}0.96^{*} \\
(0.42)\end{array}$ \\
rgdp60 & $\begin{array}{c}0.00 \\
(0.00)\end{array}$ & \\
& & $0.25^{* *}$ \\
yearsschool & & $(0.09)$ \\
\hline $\mathrm{R}^{2}$ & 0.00 & 0.11 \\
Adj. R & -0.01 & 0.10 \\
Num. obs. & 65 & 65 \\
RMSE & 1.91 & 1.80 \\
\hline${ }^{* * *} p<0.001,{ }^{* *} p<0.01,{ }^{*} p<0.05$ &
\end{tabular}

Table: Growth and GDP and education in 1960 


\section{Caution: multiple testing}

- We just looked at 6 regressions, if $H_{0}: \beta_{1}=0$ is true in all of them the probability that correctly fail to reject all 6 null hypotheses with a $5 \%$ test is $0.95^{6}=0.74$ (assuming the 6 tests are independent)

- A quarter of the time if we look at 6 regressions, we will randomly find at least significant relationship; if we look at 14 regressions the probability that we incorrectly reject a null is more than 0.5 
Introduction

to regression

Paul Schrimpf

Fitted value

and residuals

Statistical

properties

Unbiased

Variance

Distribution

Discussion of

assumptions

Examples

Inference

Examples (continued) Estimating $\sigma_{f}^{2}$

Confidence intervals

Efficiency

References

\section{Caution: economic significance $\neq$ statistical significance}




\section{Estimating $\sigma_{\epsilon}^{2}$}

- Recall that $\operatorname{Var}\left(\hat{\beta} \mid x_{1}, \ldots, x_{n}\right)=\frac{\sigma_{\epsilon}^{2}}{\sum_{i=1}^{n}\left(x_{i}-\bar{x}\right)^{2}}=\frac{\sigma_{\epsilon}^{2}}{n \operatorname{Var}(x)}$

- $\sigma_{\epsilon}^{2}$ unknown

- We estimate $\sigma_{\epsilon}^{2}$ using the residuals,

$$
\hat{\sigma}_{\epsilon}^{2}=\frac{1}{n-2} \sum_{i=1}^{n} \underbrace{\hat{\epsilon}_{i}^{2}}_{=\left(y_{i}-\hat{\beta}_{0}-\hat{\beta}_{1} x_{i}\right)^{2}}
$$

- If SLR.1-SLR.5, $\mathrm{E}\left[\hat{\sigma}_{\epsilon}^{2}\right]=\sigma_{\epsilon}^{2}$

- Using $\frac{1}{n-2}$ instead of $\frac{1}{n}$ makes $\hat{\sigma}_{\epsilon}^{2}$ unbiased

- $\hat{\epsilon}_{i}$ depends on 2 estimated parameters, $\hat{\beta}_{0}$ and $\hat{\beta}_{1}$, so only $n-2$ degrees of freedom

- Estimate $\operatorname{Var}\left(\hat{\beta}_{1} \mid x_{1}, \ldots, x_{n}\right)$ by

$$
\widehat{\operatorname{Var}}\left(\hat{\beta}_{1} \mid x_{1}, \ldots, x_{n}\right)=\frac{\hat{\sigma}_{\epsilon}^{2}}{\sum_{i=1}^{n}\left(x_{i}-\bar{x}\right)^{2}}=\frac{\frac{1}{n-2} \sum_{i=1}^{n} \hat{\epsilon}_{i}^{2}}{\sum_{i=1}^{n}\left(x_{i}-\bar{x}\right)^{2}}
$$




\section{Estimating $\sigma_{\epsilon}^{2}$}

- Standard error of $\hat{\beta}_{1}$ is $\sqrt{\widehat{\operatorname{Var}}\left(\hat{\beta}_{1} \mid x_{1}, \ldots, x_{n}\right)}$

- If SLR.1-SLR.6, t-statistic with estimated $\widehat{\operatorname{Var}}\left(\hat{\beta}_{1} \mid x_{1}, \ldots, x_{n}\right)$ has a $t(n-2)$ distribution instead of $N(0,1)$

$$
t=\frac{\hat{\beta}_{1}-\beta_{1}}{\sqrt{\widehat{\operatorname{Var}}\left(\hat{\beta}_{1} \mid x_{1}, \ldots, x_{n}\right)}} \sim t(n-2)
$$




\section{Confidence intervals}

- $\hat{\beta}_{1}$ is random

- $\widehat{\operatorname{Var}}\left(\hat{\beta}_{1}\right), \mathrm{p}$-values, and hypthesis tests are ways of expressing how random is $\hat{\beta}_{1}$

- Confidence intervals are another

- A $1-\alpha$ confidence interval, $C l_{1-\alpha}=\left[L B_{1-\alpha}, U B_{1-\alpha}\right]$ is an interval estimator for $\beta_{1}$ such that

$$
\mathrm{P}\left(\beta_{1} \in C l_{1-\alpha}=1-\alpha\right)
$$

( $C l_{1-\alpha}$ is random; $\beta_{1}$ is not)

- Recall: if SLR.1-SLR.6, then

$$
\hat{\beta}_{1} \sim N\left(\beta_{1}, \operatorname{Var}\left(\hat{\beta}_{1}\right)\right)
$$




\section{Confidence intervals}

- Implies

$$
\begin{aligned}
& \mathrm{P}\left(\hat{\beta}_{1}<\beta_{1}+\sqrt{\operatorname{Var}\left(\hat{\beta}_{1}\right)} \Phi^{-1}(\alpha / 2)\right)=\alpha / 2 \\
& \left.\mathrm{P}\left(\hat{\beta}_{1}-\sqrt{\operatorname{Var}\left(\hat{\beta}_{1}\right)} \Phi^{-1}(\alpha / 2)<\beta_{1}\right)\right)=\alpha / 2
\end{aligned}
$$

and

$$
\begin{aligned}
& \mathrm{P}\left(\hat{\beta}_{1}>\beta_{1}+\sqrt{\operatorname{Var}\left(\hat{\beta}_{1}\right)} \Phi^{-1}(1-\alpha / 2)\right)=\alpha / 2 \\
& \mathrm{P}\left(\hat{\beta}_{1}-\sqrt{\operatorname{Var}\left(\hat{\beta}_{1}\right)} \Phi^{-1}(1-\alpha / 2)>\beta_{1}\right)=\alpha / 2
\end{aligned}
$$




\section{Confidence intervals}

so

$$
\begin{aligned}
& P\left(\begin{array}{c}
\hat{\beta}_{1}+\sqrt{\operatorname{Var}\left(\hat{\beta}_{1}\right)} \Phi^{-1}(\alpha / 2)<\beta_{1} \\
\beta_{1}<\hat{\beta}+\sqrt{\operatorname{Var}\left(\hat{\beta}_{1}\right)} \Phi^{-1}(1-\alpha / 2)
\end{array}\right)= \\
= & 1-\mathrm{P}\left(\hat{\beta}_{1}+\sqrt{\operatorname{Var}\left(\hat{\beta}_{1}\right)} \Phi^{-1}(\alpha / 2)<\beta_{1}\right)- \\
& -\mathrm{P}\left(\hat{\beta}_{1}+\sqrt{\operatorname{Var}\left(\hat{\beta}_{1}\right)} \Phi^{-1}(1-\alpha / 2)>\beta_{1}\right) \\
= & 1-\alpha
\end{aligned}
$$

- For $\alpha=0.05, \Phi^{-1}(0.025) \approx-1.96, \phi^{-1}(0.975) \approx 1.96$

- For $\alpha=0.1, \Phi^{-1}(0.05) \approx-1.64$ 
Introduction

to regression

Paul Schrimpf

Fitted value

and residuals

Statistical

properties

Unbiased

Variance

Distribution

Discussion of

assumptions

Examples

Inference

Examples (continued) Estimating $\sigma_{t}^{2}$

Confidence intervals

Efficiency

References

\section{Confidence intervals}

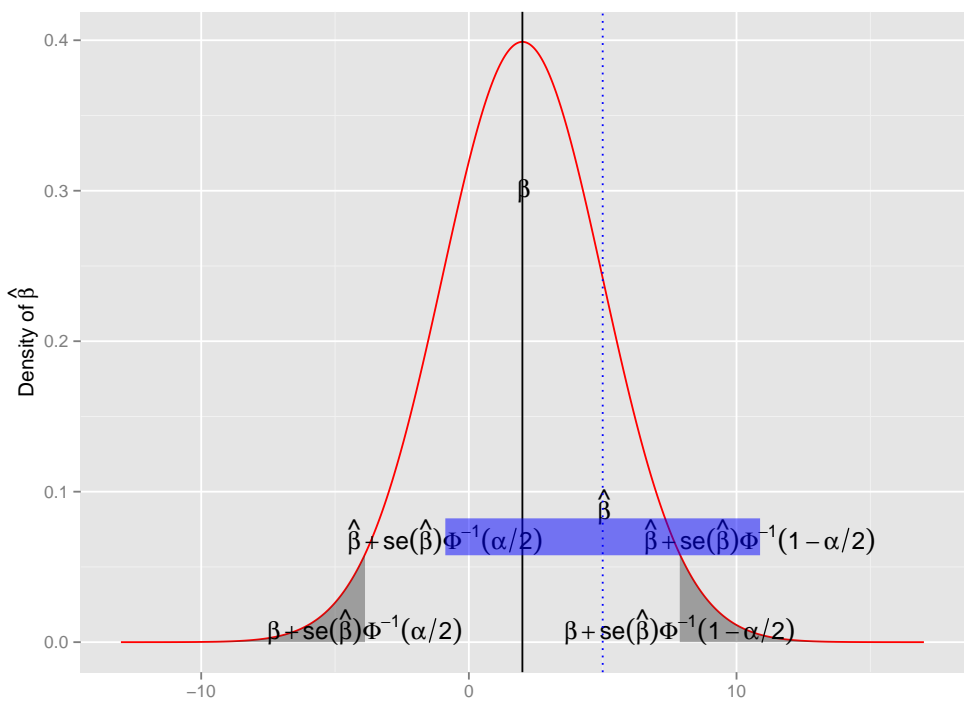




\section{Confidence intervals}

- $1-\alpha$ confidence interval

$$
\hat{\beta}_{1} \pm \sqrt{\operatorname{Var}\left(\hat{\beta}_{1}\right)} \Phi^{-1}(\alpha / 2)
$$

- With estimated $\hat{\sigma}_{\epsilon}^{2}$, use $t$ distribution instead of normal

$$
\hat{\beta}_{1} \pm \sqrt{\widehat{\operatorname{Var}}\left(\hat{\beta}_{1}\right)} F_{t, n-2}^{-1}(\alpha / 2)
$$

$F_{t, n-2}^{-1}=$ inverse CDF of $t(n-2)$ distribution

\begin{tabular}{c|cccccc} 
& \multicolumn{7}{|c}{$F_{t, n-2}(1-\alpha / 2)$} \\
$\alpha / 2$ & 5 & 10 & 20 & 50 & 100 & $\infty$ \\
\hline 0.025 & 2.57 & 2.23 & 2.09 & 2.01 & 1.98 & 1.96 \\
0.05 & 2.02 & 1.81 & 1.72 & 1.68 & 1.66 & 1.64 \\
\hline
\end{tabular}




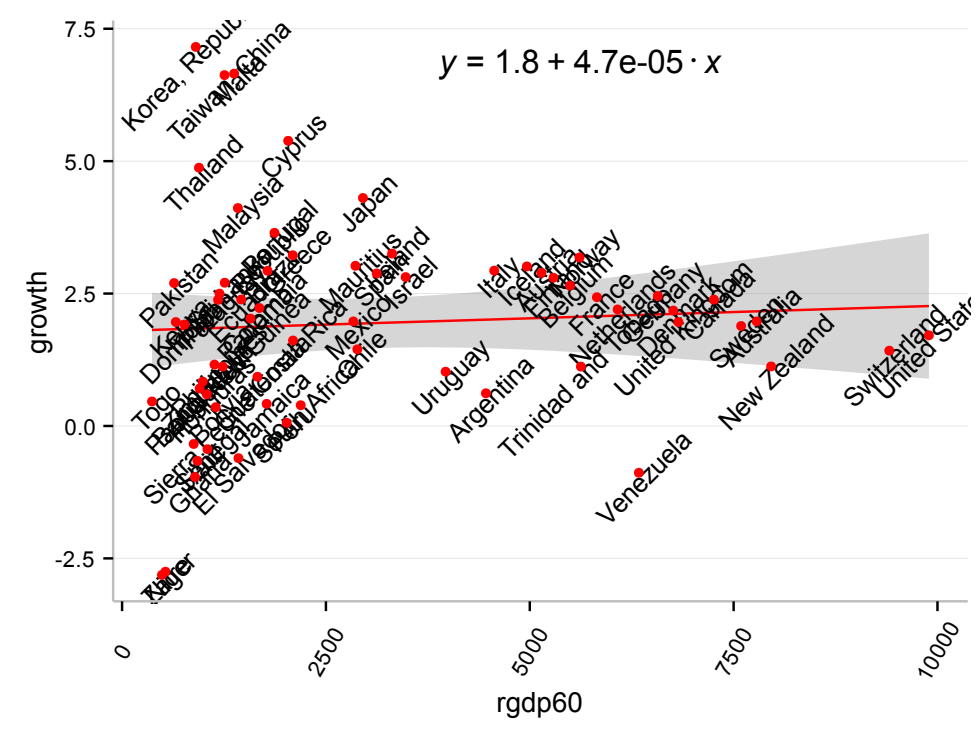

\section{Example: GDP in 1960 and} growth 


\section{Statistical}

properties

Unbiased

Variance

Distribution

Discussion of

assumptions

Examples

Inference

Examples (continued) Estimating $\sigma_{?}^{2}$

Confidence intervals

Efficiency

\section{Example: Years of schooling in 1960 and growth}

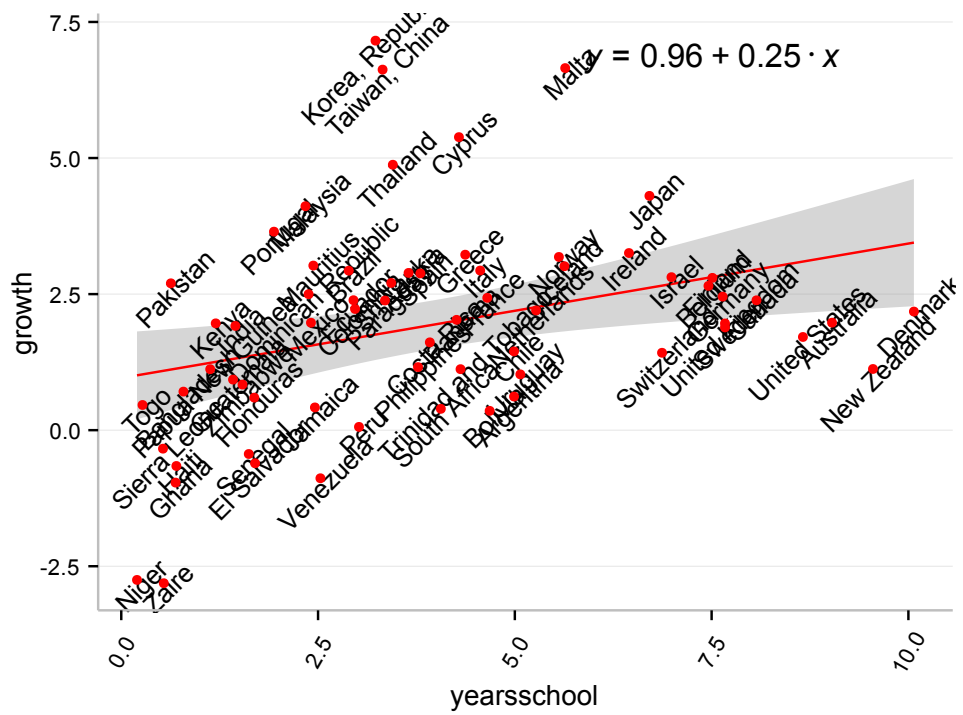


Introduction

to regression

Paul Schrimpf

Fitted value

and residuals

Statistical

properties

Unbiased

Variance

Distribution

Discussion of

assumptions

Examples

Inference

Examples (continued)

Estimating $\sigma_{f}^{2}$

Confidence intervals

Efficiency

References

\title{
Section 10
}

\author{
Efficiency
}




\section{Gauss-Markov theorem}

- The sample regression estimator,

$$
\begin{aligned}
\left(\hat{\beta}_{0}, \hat{\beta}_{1}\right) & =\arg \min \sum_{i=1}^{n}\left(y_{i}-b_{0}-b_{1} x_{i}\right)^{2} \\
\hat{\beta}_{1} & =\frac{\sum_{i=1}^{n}\left(x_{i}-\bar{x}\right) y_{i}}{\sum_{i=1}^{n}\left(x_{i}-\bar{x}\right)^{2}}
\end{aligned}
$$

also called Ordinary Least Squares (OLS) is not the only unbiased estimator of

$$
y_{i}=\beta_{0}+\beta_{1} x_{i}+\epsilon_{i}
$$

- Gauss-Markov theorem: if SLR.1-SLR.5, then OLS is the Best Linear Unbiased Estimator

- Linear means linear in $y, \hat{\beta}_{1}=\sum_{i=1}^{n} c_{i} y_{i}$ with

$$
c_{i}=\frac{\left(x_{i}-\bar{x}\right)}{\sum_{i=1}^{n}\left(x_{i}-\bar{x}\right)^{2}}
$$

- Unbiased means $\mathrm{E}\left[\hat{\beta}_{1}\right]=\beta_{1}$

- Best means that among all linear unbiased estimators, OLS has the smallest variance 


\section{Proof: setup}

- Let $\tilde{\beta}_{1}$ be a linear unbiased estimator of $\beta_{1}$

- Linear: $\tilde{\beta}_{1}=\sum_{i=1}^{n} c_{i} y_{i}$

- Unbiased: $\mathrm{E}\left[\tilde{\beta}_{1} \mid x_{1}, \ldots, x_{n}\right]=\beta_{1}$ (for all possible $\beta_{0}, \beta_{1}$ )

- We will show that

$$
\operatorname{Var}\left(\tilde{\beta}_{1} \mid x_{1}, \ldots, x_{n}\right) \geq \operatorname{Var}\left(\hat{\beta}_{1} \mid x_{1}, \ldots, x_{n}\right)
$$




\section{Proof: outline}

(1) Show that $\sum_{i=1}^{n} c_{i}=0$ and $\sum_{i=1}^{n} c_{i} x_{i}=1$

2. Show $\operatorname{Cov}\left(\tilde{\beta}_{1}, \hat{\beta}_{1} \mid x_{1}, \ldots, x_{n}\right)=\operatorname{Var}\left(\hat{\beta}_{1} \mid x_{1}, \ldots, x_{n}\right)$

3 Show $\operatorname{Var}\left(\tilde{\beta}_{1} \mid x_{1}, \ldots, x_{n}\right) \geq \operatorname{Var}\left(\hat{\beta}_{1} \mid x_{1}, \ldots, x_{n}\right)$

(4) Show $\operatorname{Var}\left(\tilde{\beta}_{1} \mid x_{1}, \ldots, x_{n}\right)=\operatorname{Var}\left(\hat{\beta}_{1} \mid x_{1}, \ldots, x_{n}\right)$ only if $\tilde{\beta}_{1}=\hat{\beta}_{1}$

${ }^{0}$ We will go over the proof in class. See Marmer's slides or Wooldridge (2013) 3A for details 


\section{References}

Abbring, Jaap. 2001. "An Introduction to Econometrics: Lecture notes." URL http://jabbring.home.xs4all.nl/ courses/b44old/lect210.pdf.

Angrist, J.D. and J.S. Pischke. 2009. Mostly harmless econometrics: An empiricist's companion. Princeton University Press.

Angrist, Joshua D and Jörn-Steffen Pischke. 2014. Mastering 'Metrics: The Path from Cause to Effect. Princeton University Press.

Baltagi, BH. 2002. Econometrics. Springer, New York. URL http:

//gw2jh3xr2c.search. serialssolutions.com/?sid= sersol\&SS_jc=TC0001086635\&title=Econometrics. 


\section{References}

Beck, T., R. Levine, and N. Loayza. 2000. "Finance and the Sources of Growth." Journal of financial economics 58 (1):261-300. URL http://www. sciencedirect.com/ science/article/pii/S0304405X00000726.

Bierens, Herman J. 2012. "The Two-Variable Linear Regression Model." URL http://personal.psu.edu/hxb11/LINREG2.PDF.

Diez, David M, Christopher D Barr, and Mine Cetinkaya-Rundel. 2012. OpenIntro Statistics. OpenIntro. URL http://www.openintro.org/stat/textbook.php.

Linton, Oliver B. 2017. Probability, Statistics and Econometrics. Academic Press. URL

http://gw2jh3xr2c.search.serialssolutions.com/ ?sid=sersol\&SS_jc=TC0001868500\&title= Probability $\% 2 \mathrm{C} \% 20$ statistics $\% 20$ and $\% 20$ econometrics. 


\section{References}

Stock, J.H. and M.W. Watson. 2009. Introduction to Econometrics, 2/E. Addison-Wesley.

Wooldridge, J.M. 2013. Introductory econometrics: A modern approach. South-Western. 